\title{
Localization of rf breakdowns in a standing wave cavity
}

\author{
Faya Wang and Chris Adolphsen \\ SLAC, Menlo Park, California 94025, USA \\ (Received 4 February 2009; published 21 April 2009)
}

\begin{abstract}
At SLAC, a five-cell, normal-conducting, $L$-band $(1.3 \mathrm{GHz})$, standing-wave (SW) cavity was built as a prototype positron capture accelerator for the ILC. The structure met the ILC gradient goal but required extensive rf processing. When rf breakdowns occurred, a large variation was observed in the decay rate of the stored energy in the cavity after the input power was shut off. It appeared that the breakdowns were isolating sections of the cavity, and that the trapped energy in those sections was then partitioned among its natural modes, producing a distinct beating pattern during the decay. To explore this phenomenon further, an equivalent circuit model of cavity was created that reproduces well its normal operating characteristics. The model was then used to compute the spectra of trapped energy for different numbers of isolated cells. The resulting modal patterns agree well with those of the breakdown data, and thus such a comparison appears to provide a means of identifying the irises on which the breakdowns occurred.
\end{abstract}

DOI: 10.1103/PhysRevSTAB.12.042001

PACS numbers: 29.20.Ej, 52.70.Gw

\section{INTRODUCTION}

Although the ILC uses predominately superconducting $L$-band $(1.3 \mathrm{GHz})$ cavities, the capture cavity that follows the positron target needs to be normal conducting due to the high radiation levels and the need for a solenoidal magnetic field for focusing. A half-length (five-cell) prototype standing-wave cavity was built at SLAC to verify that the relatively high gradient $(15 \mathrm{MV} / \mathrm{m})$ required for efficient capture can be reliably achieved with the long $(1.0 \mathrm{~ms})$ ILC pulses [1-4]. Figure 1 shows a cross section of the cavity, whose design was complicated by the extensive cooling required to prevent significant detuning from average rf heating during its $5 \mathrm{~Hz}$ operation.

Although the cavity reached the design gradient, it incurred several thousand breakdowns during rf processing. To study these events, transients digitizers were used to record the input power, reflected power, and stored energy in the cavity during the breakdown pulses. The stored energy was measured with an $\mathrm{rf}$ probe located in the beam pipe just after the last cell at the downstream end of the cavity (i.e., the end opposite of the power coupler). After a breakdown, which was detected by an increase in reflected power or a drop in stored energy, the input power was shut off within about $1 \mu \mathrm{s}$. Interestingly, the subsequent decay times of the stored energy varied greatly from event to event, taking from a few $\mu$ s to over $15 \mu$ s to decrease by $20 \mathrm{~dB}$. Also, there appeared to be a beating pattern during the decays, all of which suggests that the breakdowns (probably on the irises) were causing the rf energy to be isolated in the downstream cells, and that the trapped energy was being partitioned among the resulting modes of those cells. This prompted a study of the cavity modes with an equivalent circuit model to see if the predicted decay spectra for various numbers of isolated down- stream cells match the data spectra, thus identifying the likely irises on which the breakdowns had occurred.

This paper is organized as follows. In Sec. II, an equivalent circuit model for a multicell SW cavity is developed, and in Sec. III, the static and transient properties of the ILC cavity predicted by this model are compared with measurements. A comparison is then presented in Sec. IV between the model's prediction for the decay spectra peaks
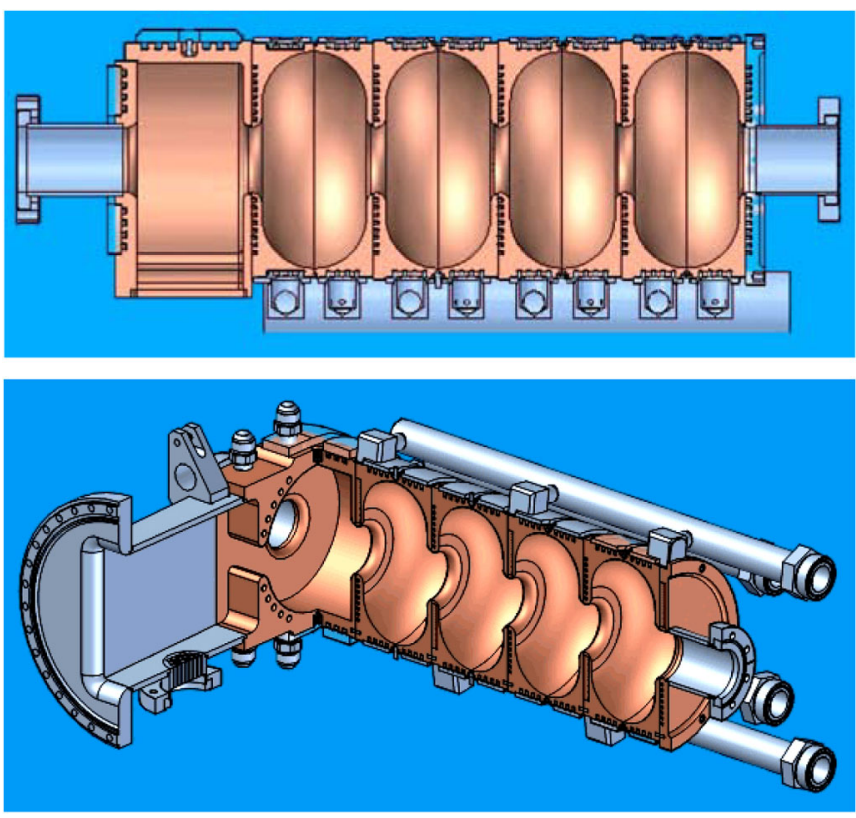

FIG. 1. (Color) Cross sectional view of the five-cell cavity where the coupler cell is on the left and an rf probe (not shown) is located in the beam pipe just outside the rightmost cell. Cooling water circulates through rectangular grooves in the irises and outer cavity walls. 
and those measured in $\mathrm{rf}$ breakdown events. Finally, a discussion and summary is given in Sec. V.

\section{COUPLED RESONATOR EQUATIONS FOR A MULTICELL SW CAVITY}

For a SW multicell cavity with coupling at one end (cell 1), a single-band equivalent circuit model is shown in Fig. 2. Based on this model, the time-dependent circuit equation for the $n$th cell $(n \geq 2)$ is

$$
\begin{aligned}
& \frac{d^{2} \hat{v}_{n}}{\omega_{n}^{2} d t^{2}}+\frac{1}{\omega_{n} Q_{n}} \frac{d \hat{v}_{n}}{d t}+\left(\frac{1}{1-k_{n-1, n}^{2}}+\frac{1}{1-k_{n, n+1}^{2}}\right) \frac{\hat{v}_{n}}{2} \\
& =\frac{1}{2}\left[\frac{k_{n-1, n}}{1-k_{n-1, n}^{2}} \sqrt{\frac{(R / Q)_{n} \omega_{n-1}}{(R / Q)_{n-1} \omega_{n}}} \hat{v}_{n-1}\right. \\
& \left.\quad+\frac{k_{n+1, n}}{1-k_{n+1, n}^{2}} \sqrt{\frac{(R / Q)_{n} \omega_{n+1}}{(R / Q)_{n+1} \omega_{n}}} \hat{v}_{n+1}\right],
\end{aligned}
$$

where $\hat{v}_{n}=\sqrt{C_{n}} V_{n}$ is the normalized $n$th cell voltage. Also, the time-dependent circuit equation for the drive cell (with input coupler) is

$$
\begin{aligned}
& \frac{d^{2} \hat{v}_{1}}{\omega_{1}^{2} d t^{2}}+\frac{1}{\omega_{1} Q_{1}} \frac{d \hat{v}_{1}}{d t}+\left(1+\frac{1}{1-k_{1,2}^{2}}\right) \frac{\hat{v}_{1}}{2} \\
& =\frac{k_{1,2}}{2\left(1-k_{1,2}^{2}\right)} \sqrt{\frac{(R / Q)_{1} \omega_{2}}{(R / Q)_{2} \omega_{1}}} \hat{v}_{2}+\frac{d}{\omega_{1} d t}\left(\hat{I}_{g}-\frac{\hat{v}_{1}}{\hat{R}_{g} Q_{1}}\right),
\end{aligned}
$$

where $\hat{I}_{g}$ is the normalized drive current, $\hat{I}_{g}=\sqrt{L_{1}} I_{g}$, and
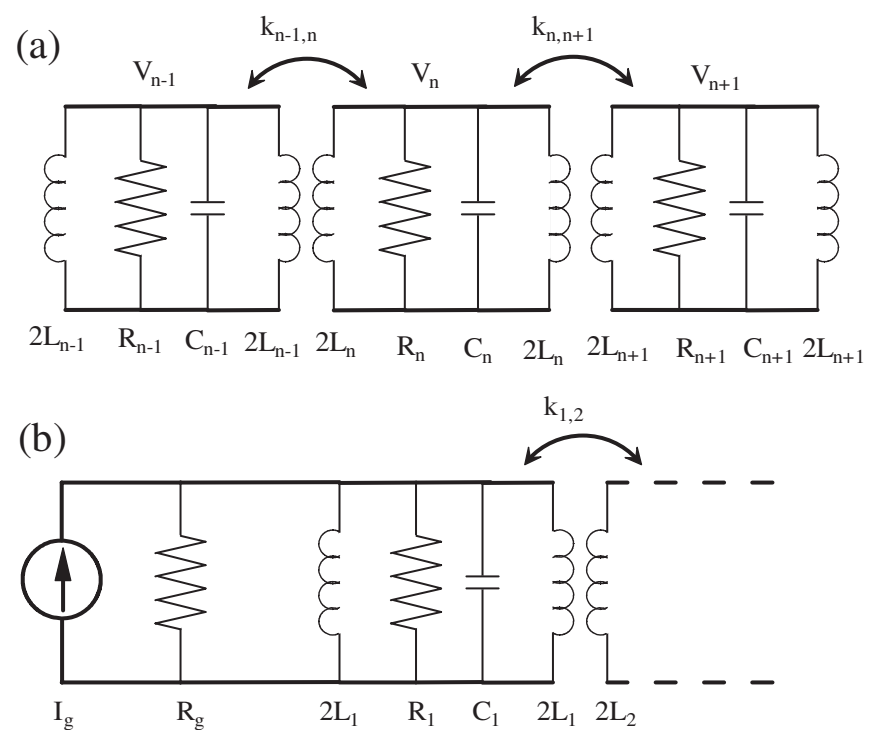

FIG. 2. General equivalent circuit model for a SW cavity powered from one end. Diagram (a) shows the regular cell equivalent circuit where $k$ is the coupling coefficient between neighboring cells, and diagram (b) shows the input coupler cell and the power generator equivalent circuit, where $R_{g}$ is the generator impedance.
$\hat{R}_{g}$ is the normalized generator impedance, $\hat{R}_{g}=$ $R_{g} /\left(R_{1} / Q_{1}\right)$.

For a uniform $N$-cell SW cavity driven at the $q$ th mode frequency, it can be shown from energy conservation that the cavity impedance, $Z_{\text {cav }}$, reduces to a real impedance, $R_{q}$, such that $R_{q}=R V_{1}^{2}\left(\phi_{q}\right) / \sum_{n=1}^{N} V_{n}^{2}\left(\phi_{q}\right)$, where $R$ is the single cell shunt impedance, $\phi_{q}$ is the phase advance per cell, and $V_{n}\left(\phi_{q}\right)(n=1 \ldots N)$ is the $n$th cell voltage in the $q$ th mode. The coupling to the cavity, $\beta_{q} \equiv R_{q} / R_{g}$, can then be expressed as

$$
\beta_{q}=\frac{R}{R_{g}} \frac{V_{1}^{2}\left(\phi_{q}\right)}{\sum_{n=1}^{N} V_{n}^{2}\left(\phi_{q}\right)} .
$$

By solving the coupled differential equations [Eqs. (1) and (2)] with the cavity coupling constraint [Eq. (3)], the transient response of a uniform multicell SW cavity can be determined.

\section{CAVITY PARAMETERS}

The $\pi$-mode coupling, $\beta_{\pi}$, of the ILC cavity was determined from the on-frequency, steady-state reflection measured during high power operation. The reflection was about $-30 \mathrm{~dB}$ so $\beta_{\pi}$ is within $6 \%$ of the unity design value. For the remainder of this paper, $\beta_{\pi}$ will be approximated to be unity as this has little effect on the results presented. The coupling for the other modes are listed in Table I and were computed by scaling from $\beta_{\pi}=1$ using Eq. (3) with $V_{n}\left(\phi_{q}\right)$ proportional to $\sin \left\lfloor(n-1 / 2) \phi_{q}\right\rfloor$, where $\phi_{q}=\pi q / N$ and $q=1,2, \ldots N$ [5]. These are the relative field values expected given the end-cell boundary conditions for the ILC cavity.

Table I also lists cold test measurements of the mode frequencies that were made before the cavity was operated at high power [6]. To determine the cell-to-cell coupling, $k$, the mode frequencies were fit to the form, $f_{q}^{2}=f_{0}^{2} /(1+$ $k \cos \phi)$, which yields $k=0.013$. Of the remaining three circuit parameters for the non-end cells ( $L, C$, and $R$ ), only two need to be specified. Choosing $R=1$, and using the measured values of the $\pi$-mode frequency, $f_{\pi}$, and $Q_{L}$ from high power measurements, the values of $L=$ $1 /\left(2 Q_{L} \omega_{0}\right)$ and $C=2 Q_{L} / \omega_{0}$ were determined where $\omega_{0}=2 \pi f_{\pi} \sqrt{1-k}$. For the end cells, $R=1$ and $L$ and $C$ were computed using $\omega_{0}=2 \pi f_{\pi} \sqrt{1+k / 2} \sqrt{1-k}$ [5],

TABLE I. Approximate values of the cavity mode couplings $\left(\beta_{q}\right)$, and mode frequencies measured during cold tests.

\begin{tabular}{llc}
\hline \hline Mode & $\beta_{q}$ & Frequency \\
\hline$\pi / 5$ & 0.19 & $1285.4 \mathrm{MHz}$ \\
$2 \pi / 5$ & 0.7 & $1289.3 \mathrm{MHz}$ \\
$3 \pi / 5$ & 1.3 & $1294.3 \mathrm{MHz}$ \\
$4 \pi / 5$ & 1.8 & $1298.4 \mathrm{MHz}$ \\
$\pi$ & 1.0 & $1300.0 \mathrm{MHz}$ \\
\hline \hline
\end{tabular}




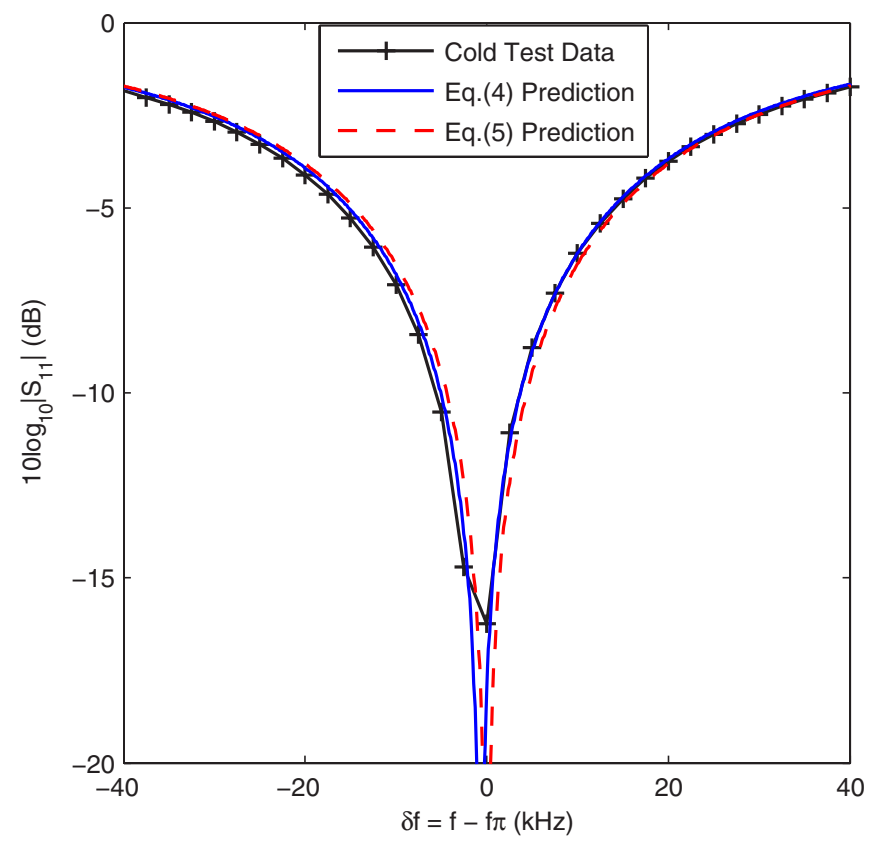

FIG. 3. (Color) Cold test measurements of the cavity reflection (S11) near the $\pi$-mode frequency and predictions from Eqs. (4) and (5).

which provides the proper boundary conditions. Finally, $R_{g}$ is $1 / 5$ with the critical coupling $\left(\beta_{\pi}=1\right)$ assumption.

\section{A. Steady-state characteristics of cavity}

The reflection coefficient $\Gamma$ of a cavity is given by

$$
\Gamma=\frac{Z_{\mathrm{cav}}-R_{g}}{Z_{\mathrm{cav}}+R_{g}}=\frac{\beta_{q} \cdot Z_{\mathrm{cav}}-R_{q}}{\beta_{q} \cdot Z_{\mathrm{cav}}+R_{q}}
$$

which to first order in $\Delta \omega$ can be written [7]

$$
\Gamma(\Delta \omega)=\frac{1-\beta_{q}-2 i \tau_{0} \Delta \omega}{1+\beta_{q}-2 i \tau_{0} \Delta \omega},
$$

where $\tau_{0}=Q_{0} / \omega_{q}, \Delta \omega=\omega-\omega_{q}$, and $\omega$ is the drive frequency. In Fig. 3, the reflection coefficient versus frequency near the $\pi$ mode that was computed from Eqs. (4) and (5) using the equivalent circuit parameters discussed above is compared with cold test measurements. Both predictions agree well with data showing that the "hot" and "cold" $Q$ 's are very similar $\left(Q_{L}=14300\right)$ and the $\pi$-mode response function is Lorentzian-like as expected. Also, the loaded $Q$ 's measured during high power operation at the $4 \pi / 5$ and $3 \pi / 5$ modes are consistent with the couplings for these modes listed in Table I.

\section{B. Transient properties of cavity}

For a cavity with widely spaced modes in comparison to the mode widths, essentially only the on-frequency mode will be excited and the cavity field will change smoothly versus time when it is being filled or discharged. But for a

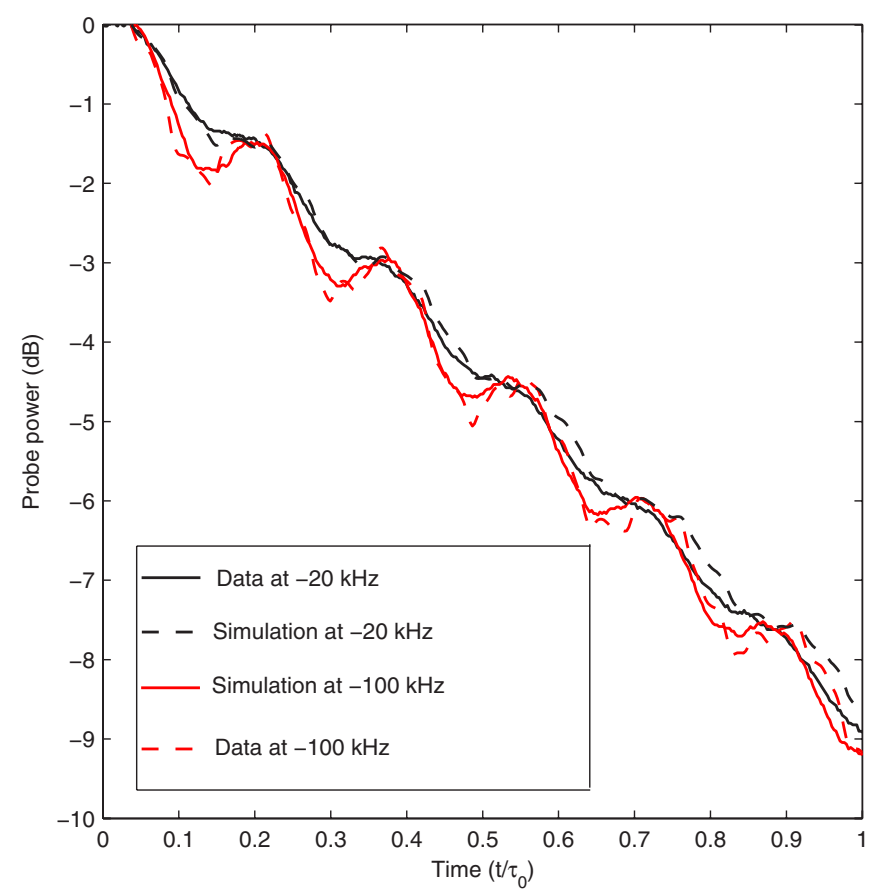

FIG. 4. (Color) Measurements and equivalent circuit model predictions of the probe power after the cavity drive is tuned off. The black and red curves are for drive frequencies 20 and $100 \mathrm{kHz}$ below the $\pi$-mode frequency, respectively.

cavity with closely spaced modes, there will be a much larger excitation of neighboring modes. This will manifest itself as a beating of the field amplitudes during the fill and discharge periods if the cavity is driven by a fast rise and fall time pulse (i.e., much shorter than the loaded $Q$ divided by mode frequency spacing).

For the five-cell, normal-conducting ILC cavity, the frequency spacing between the $\pi$ mode and the next nearest mode is about 17 times the mode width, so the beating effect is fairly small. To test the transient predictions of the equivalent circuit model, it is thus better to compare to data taken with the cavity driven off frequency to enhance the nearby mode excitations. Figure 4 shows the probe power waveform after the drive rf was shut off for drive frequencies $20 \mathrm{kHz}$ and $100 \mathrm{kHz}$ below the $\pi$ mode frequency. Also shown are the simulated results for these two cases including the effects of the $30 \mathrm{MHz}$ bandwidth filter and the $100 \mathrm{MHz}$ sampling used for the measurements. The good match provides further evidence of the validity of the model.

\section{BREAKDOWN LOCATION IN THE CAVITY}

\section{A. Cavity breakdown simulation}

Breakdowns in high voltage and rf systems are known to be accompanied by the formation of a metal ion plasma just above the surface of the breakdown site that dramatically alters the system operation [8]. For example, in tests of X-band $(11.4 \mathrm{GHz})$ traveling wave structures with few- 
hundred ns long rf pulses, breakdown is accompanied by high currents $(\sim 1 \mathrm{~A})$ and intense $\mathrm{x}$ rays while the transmitted power drops essentially to zero [9]. However, visible light (including $\mathrm{Cu}$ atom lines) continues to be emitted from the structure for several $\mu \mathrm{s}$ after the main rf pulse is shut off and much lower rf power continues to enter the structure as the SLED-based rf source fully discharges. Also the $\mathrm{rf}$ transmission through the structure remains blocked on the several $\mu$ s time scale, and then it quickly $(<1 \mu \mathrm{s})$ restores to the level that would occur without breakdown. Thus, it appears that after the initial large current and $\mathrm{x}$-ray transient, the plasma that is formed continues to affect the structure transmission properties.

Just how this happens is not clear, but the plasma expansion probably detunes the neighboring cells and reduces the coupling between cells. Measurements of ions emitted during breakdown in a $30 \mathrm{GHz}$ structure show their maximum velocity to be about $1 \times 10^{4} \mathrm{~m} / \mathrm{s}$ [10]. If the velocities are similar in the ILC cavity, then the plasma would spread out on the order of $1 \mathrm{~cm}$ in $1 \mu \mathrm{s}$, which is small compared to the $10 \mathrm{~cm}$ iris spacing but more significant compared to the $3 \mathrm{~cm}$ iris radius. So on this time scale, the largest plasma effect may be to reduce the coupling through the irises in which the breakdown occurs. This will be assumed in the following analysis, and is supported by other observations that are described below.

To simulate the effect of a breakdown on a particular iris with the equivalent circuit model, the cell-to-cell coupling $(k)$ through that iris was set to zero after the fields reached steady state in the $\pi$ mode. The model shows that the stored energy remaining in each section of the now divided

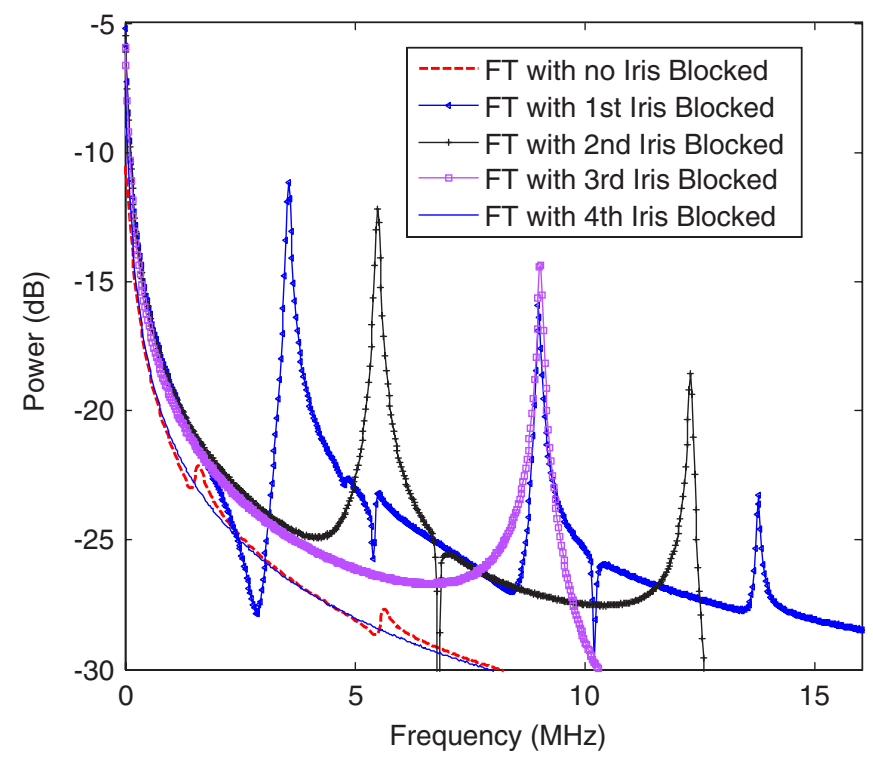

FIG. 5. (Color) Predicted frequency spectrum of the log of the probe power at the downstream end of the cavity when different irises are blocked and the cell fields are equal initially, except for the no iris blocked case, in which the first cell has a field $3 \%$ lower than the others. cavity will excite all possible modes in that section to some degree. As these modes decay, the amplitude of the store energy shows a beating pattern that depends on the frequency differences. Figure 5 shows plots of the Fourier transform (FT) of the log of the simulated probe power (no phase information is used) in the downstream section during a $10 \mu$ s period after different irises are blocked. In the case where no irises are blocked, the initial field in the first cell was assumed to be $3 \%$ lower than others to produce a noticeable beating. For other cases, the initial fields in the cells were assumed to be equal, which may not be the case just after breakdown due to the breakdown-generated currents. If the initial fields are not equal, there are other mode frequencies that may appear, and these will also be considered in the next section (for example, with four cells isolated, there are six possible frequency differences, although only three of them show pronounce peaks in the simulated FT spectrum in Fig. 5).

\section{B. Cavity breakdown data}

In Figs. 6-10, waveform data from five events are shown that were chosen as typical examples of what is interpreted as breakdowns on each of the four irises, and at a location just outside the cavity, perhaps near the window. The beam pipe transition regions in the end cells have much lower surface fields than the irises and probably do not break down (in particular, if a breakdown occurred at the downstream end transition region where the field probe is located, it would likely produce a very strong probe signal, which was not seen).

The waveform data shown in these figures were taken with a $100 \mathrm{MHz}$ sampling scope that started $(t=0)$ shortly after the main input power was shutoff (which occurred about $1 \mu$ s after a fault was detected). Both the reflected and probe power are normalized to $0 \mathrm{~dB}$ at this point, as are these values for the nonbreakdown event that are shown with dashed lines in the figures (in this case, the two curves track each other as expected). For the breakdown events, the reflected power decreases very fast, but has a profile that is complicated by the fact that the input power was first reduced by about $20 \mathrm{~dB}$ before $t=0$ and then was fully shut off a few $\mu$ s later through a different mechanism. In Fig. 6(a), for example, there is a "ledge" in the reflected power curve at about $-20 \mathrm{~dB}$ for a few $\mu$ s before it falls to the measurement noise level at around $-35 \mathrm{~dB}$. In contrast, the probe power, which is a measure of stored energy, decreases relatively slowly, and shows that the breakdown was effective at isolating the downstream portion of the cavity. In fact, the probe power decay rate is about half the nominal rate (i.e., roughly consistent with $Q_{0}$ ) so the isolating mechanism does not itself absorb much power (although for the other examples, it does decrease faster). The observed ratio of probe-to-reflected power provides a rough measure of the effective reduction in the cell coupling $(k)$ caused by breakdown. To match the maximum 

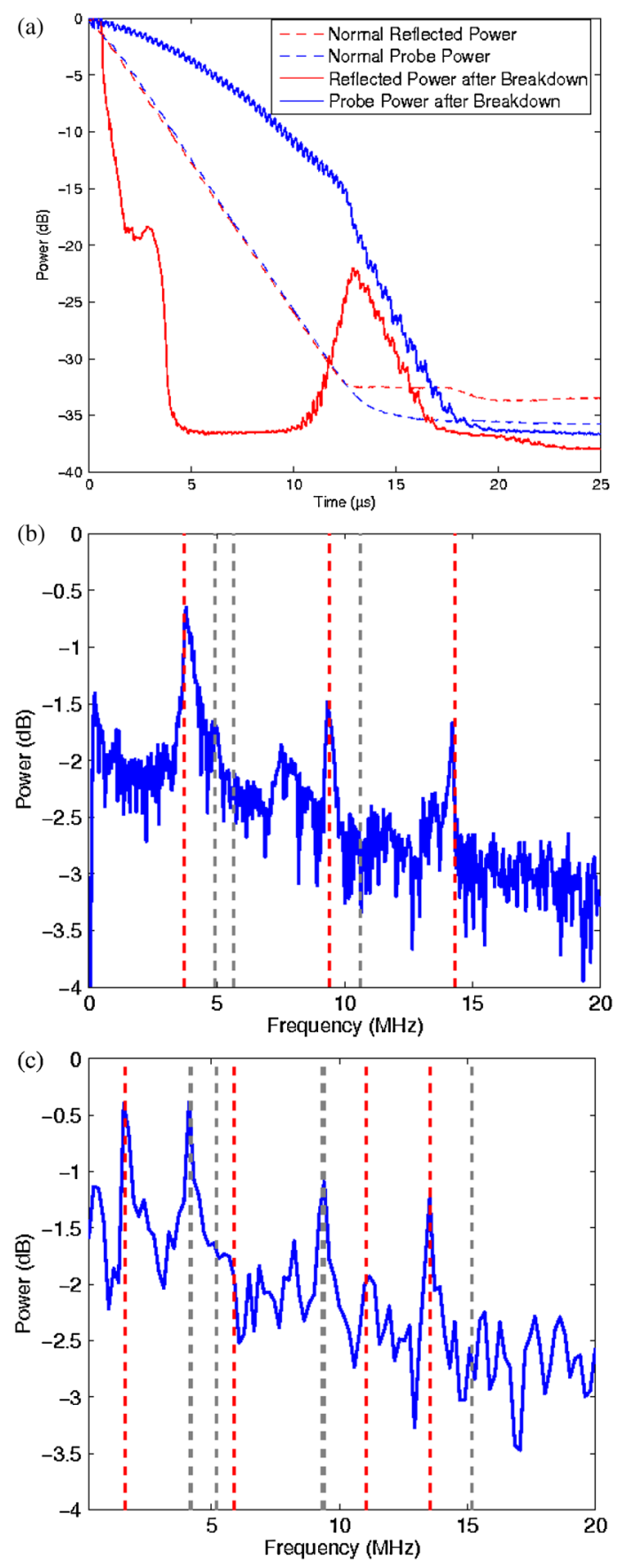

FIG. 6. (Color) (a) The blue and red-dashed lines are the probe power and reflected power, respectively, when the cavity discharges at the end of the pulse (no breakdown), and the solid lines are these waveforms for a breakdown event where the time scale has been shifted so $t=0$ aligns with the beginning of the normal discharge. (b) The FT of the log of the probe power in the $0-12 \mu$ s time range (blue solid line), and the peak spectral frequencies in the simulated measurement in the case where the first iris is blocked (the vertical red lines correspond to the resulting peak frequencies when the initial fields are equal in each cell when $k$ is zeroed, and the grey vertical lines are the other possible beating frequencies). (c) Same as (b) but for the 12-18 $\mu$ s data, and the case where no irises are blocked in the simulation but only the downstream four cells are filled initially.
$20 \mathrm{~dB}$ ratio that occurs when the reflected power is noise dominated, $k$ in the circuit model needs to be reduced by 95\%. Thus, the effective reduction is even higher and zeroing $k$ in the circuit model is a good approximation.

Another interesting feature of the data in Fig. 6 is the eventual turn on the reflected power that then tracks the decay of the probe power after $t=13 \mu \mathrm{s}$. The mechanism that was isolating the stored energy appears to be no longer effective and so the energy flows out through the coupler and appears as reflected power. The time scale for this transition is similar to that observed in X-band structures as noted above, and suggests the effect is a function of the plasma only (e.g., the recombination time of the ions and electrons).
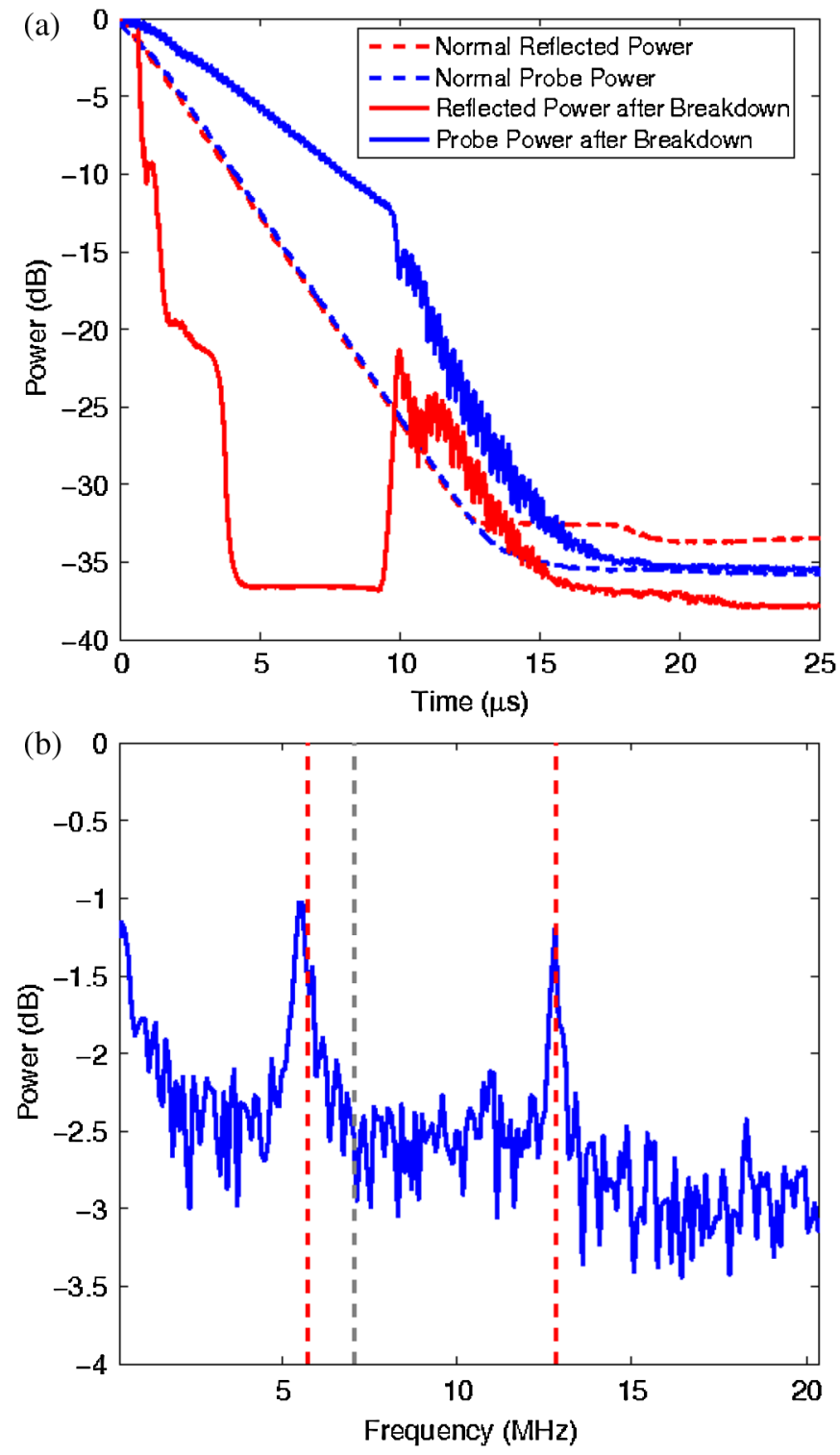

FIG. 7. (Color) (a) Same description as Fig. 6(a). (b) Same description as 6(b) but for the 0-9 $\mu$ s data, and the case where the second iris is blocked in the simulation. 

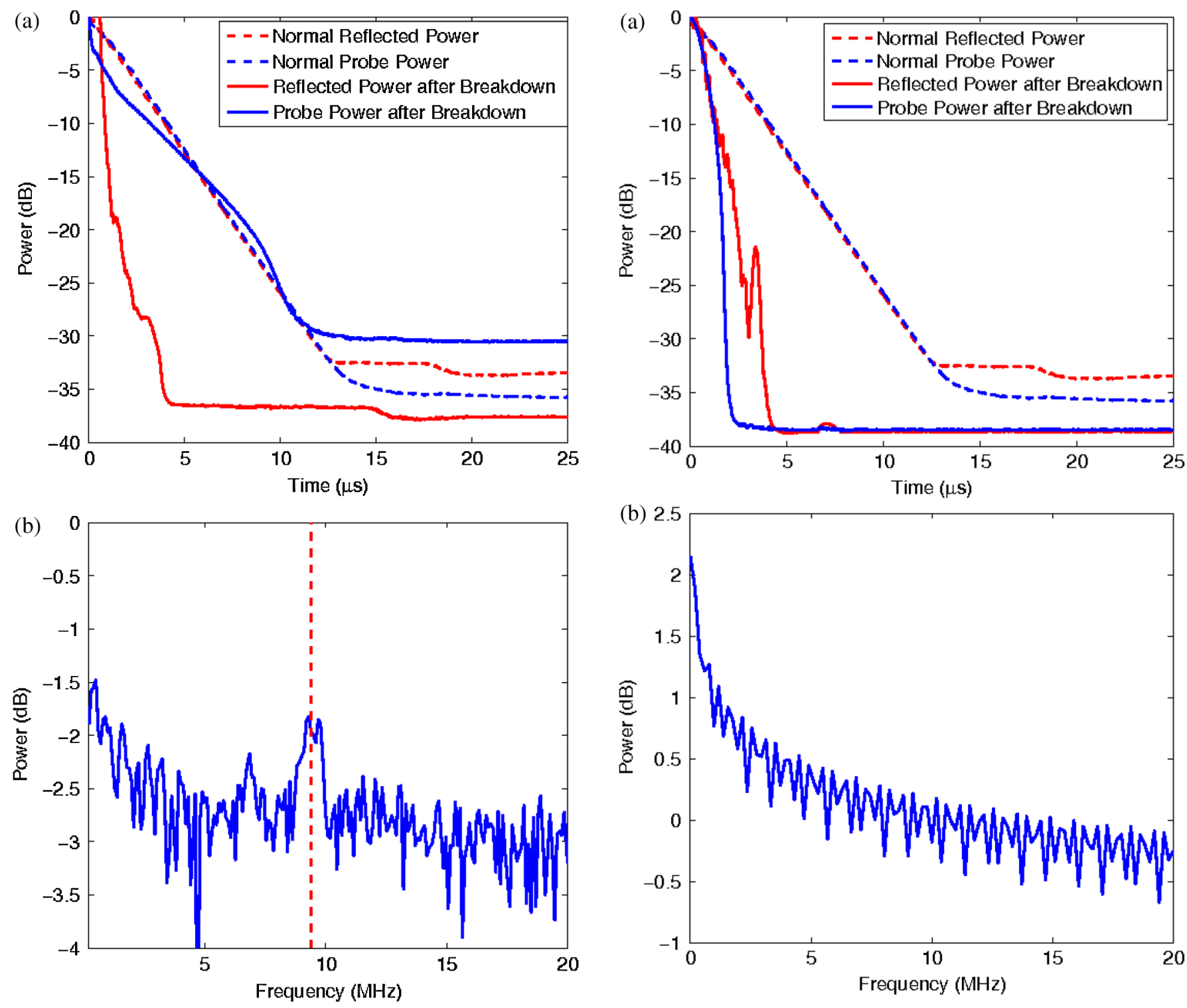

FIG. 8. (Color) (a) Same description as Fig. 6(a). (b) Same as description 6(b) but for the 0-9 $\mu$ s data, and the case where the third iris is blocked in the simulation.

Figures 6-10 also include plots of the FT of the log of the probe power computed over the time ranges noted in the figure captions. For example, Fig. 6(b) is the FT of the log of the probe power from $0-12 \mu \mathrm{s}$, and includes vertical lines corresponding to the spectral peaks predicted in the case when the first iris is blocked. The red-dashed vertical lines are those corresponding to the peaks in Fig. 5 (in which it is assumed the isolated cells have equal fields initially), and the gray-dashed vertical lines are all possible additional beating frequencies. The good agreement of the data peaks with those in Fig. 5 suggests the breakdown in this case is isolating the stored energy in the downstream four cells. However, it is not clear why the data peaks are less pronounced than in the simulations (and correspondingly, the simulated probe power signal has much higher

FIG. 9. (Color) (a) Same description as Fig. 6(a). (b) Same description as 6(b) but for the $0-2 \mu$ s data, and no simulation results are included as no beating occurs if the fourth iris is blocked (i.e., only one mode is present in the fifth cell).

peak-to-valley variations than the data). In Fig. 6(c), a similar FT comparison is made, but this time with the 12-18 $\mu \mathrm{s}$ data after the reflected power turns on again. In this case, the vertical lines are for the full five-cell cavity with the downstream four cells having equal fields initially and the coupler cell having zero field initially. The match of the vertical lines to the data peaks is consistent with the unblocking of the first iris.

Figures 7(b), 8(b), and 9(b) are similar to Fig. 6(b) except that the second, third, and fourth irises, respectively, are blocked in the simulation. Again, there is good agreement between the data peaks and those in Fig. 5 for the second and third blocked iris case. For the fourth iris, since there is only one mode in the last cell, there should be no 

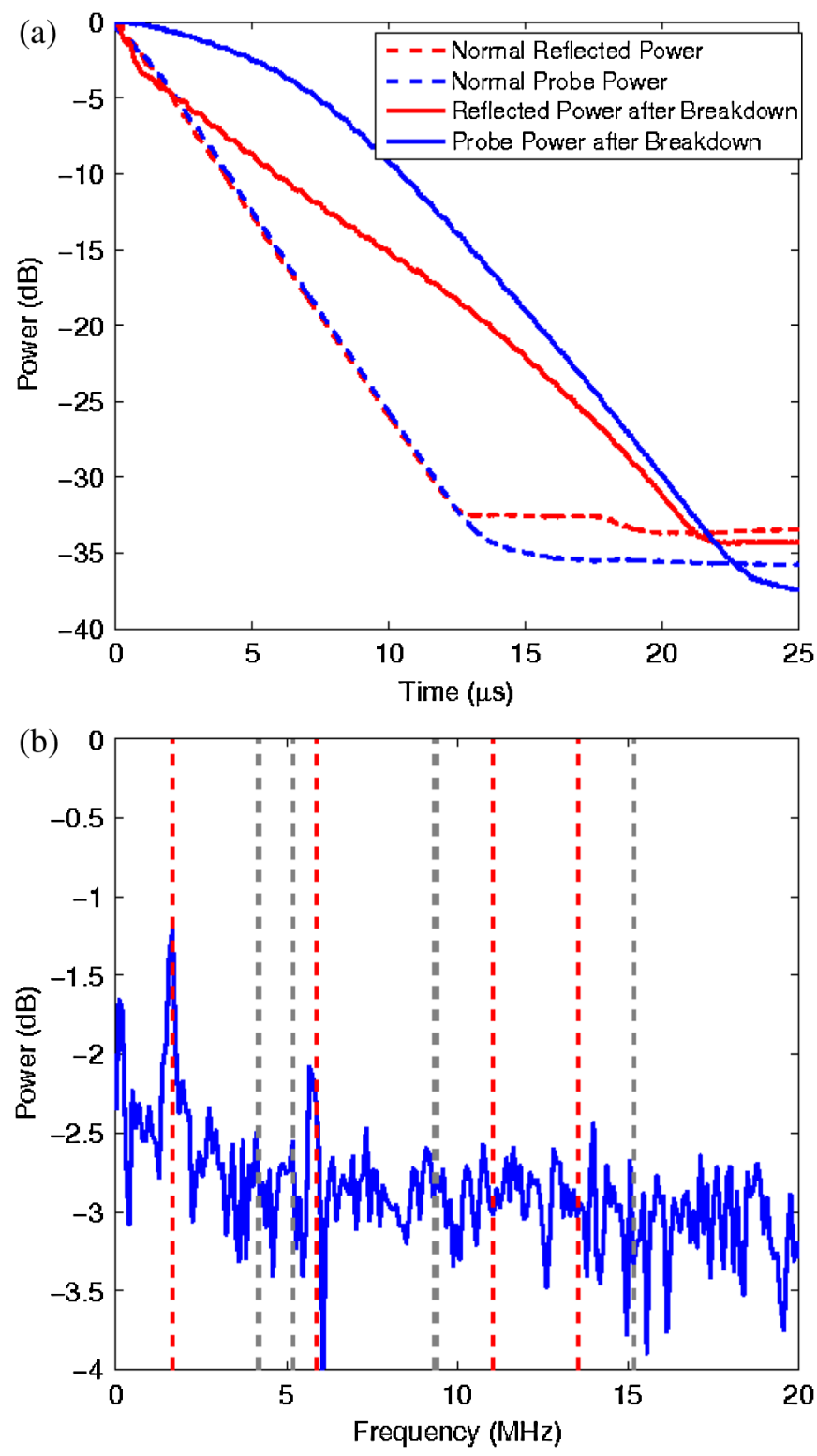

FIG. 10. (Color) (a) Same description as Fig. 6(a). (b) Same description as 6(b) but for the 0-20 $\mu$ s data, and the case where no irises are blocked and all cells have equal fields initially, except the first cell, which has a $3 \%$ lower field than the others.

beating as is observed in the data. Finally, in Fig. 10, the breakdown probably happened in the waveguide between the cavity and directional coupler that measures reflected power. In this case, the prominent data peaks match the predicted peaks in the case where no irises are blocked and all cells have equal fields initially, except the first cell, which has a $3 \%$ lower field than the others (this difference is needed to produce a noticeable beating).

All events map into one of five patterns of breakdown noted above where the FT peaks of the data match those of the predictions if the isolated cells essentially have equal fields initially. The fact that all possible iris patterns are seen supports the assumption that the breakdowns do not significantly detune the neighboring cells. As noted earlier, the reflected power decreases quickly and there is no discernible beating effect. However, the simulated reflected power decays slower than the data (although faster than the normal shutoff case) and has a pronounced beating effect, so there is more involved in the breakdowns than just the simple isolation of the downstream cells. Nonetheless, the peak probe spectra provide a unique signature that is likely indicating the iris on which the breakdown occurred.

\section{SUMMARY}

A five-cell, $1.3 \mathrm{GHz}$ cavity built as a prototype for the ILC positron capture accelerator has served as a useful test bed to explore breakdown phenomena in SW cavities. To aid this study, an equivalent circuit was established that was shown to match well particular static and transient properties of the cavity under normal operation. For breakdown events, it was assumed that the cavity is isolated at a given iris and the stored energy is then partitioned into the modes of the reduced number of cells. The peaks in the probe power spectrum from breakdown events match the patterns predicted by the model with this assumption. Thus, such a comparison appears to provide a means of localizing the breakdowns, which is generally hard to do in SW cavities. If more probes could be added to the cavities (e.g., one in each cell), perhaps the details of the plasma evolution could be better understood.

\section{ACKNOWLEDGMENTS}

We wish to thank Juwen Wang, Zenghai Li, Chris Nantista, Erik Jongewaard, Gordon Bowden, and the ARD Test Facilities Group for their efforts on the design, construction, cold testing, and high power operation of this cavity. We also thank Chris Nantista for his careful reading of this document.

[1] J. W. Wang et al., SLAC Report No. SLAC-PUB-12412, 2007.

[2] J.W. Wang et al., SLAC Report No. SLAC-PUB-11767, 2006.

[3] J.W. Wang, "Progress in NC Accelerator Structures," Report on ILC R\&D Meeting, SLAC, 2007.

[4] F. Wang, C. Adolphsen, and J. Wang, SLAC Report No. SLAC-PUB-13459, 2008.

[5] S. O. Schriber, CERN Report No. CERN/PS/2001-067 (PP).

[6] J.W. Wang (private communication).

[7] Tom Hays, SRF Report No. SRF 940525-04, 1994.

[8] J. W. Wangand and G. A. Loew, SLAC Report No. SLACPUB-4647, 1988.

[9] C. Adolphsen (unpublished).

[10] M. Johnson, R. Ruber, V. Ziemann, and H. Braun, Nucl. Instrum. Methods Phys. Res., Sect. A 595, 568 (2008). 